C. Berg

Nagoya Math. J.

Vol. 70 (1978), 157-165

\title{
ON THE EXISTENCE OF CONDENSER POTENTIALS
}

\author{
CHRISTIAN BERG
}

\section{Introduction}

The existence of condenser potentials was established in the framework of Dirichlet spaces by Beurling and Deny, cf. Deny [5] or Landkof [10], simply by choosing the potential of minimal energy within a certain convex set. This same idea works for non-symmetric Dirichlet spaces, cf. Bliedtner [3].

Let $\left(\mu_{t}\right)_{t>0}$ be a transient convolution semigroup on a locally compact abelian group $G$ and let $\kappa=\int_{0}^{\infty} \mu_{t} d t$ be the potential kernel. The associated negative definite function on $\hat{G}$ is denoted $\psi$. For an explicit formulation of these concepts see [2]. We may now state that $\kappa$ satisfies the condenser principle in the special case where $\kappa$ is associated with an ordinary Dirichlet space, corresponding to $\psi$ being realvalued, cf. [7], or more generally in the case where $\kappa$ is associated with a non-symmetric Dirichlet space, corresponding to $\psi$ satisfying the inequality $|\operatorname{Im} \psi| \leq$ $A \operatorname{Re} \psi$, cf. [1].

The purpose of this note is to show that every potential kernel $\kappa$ satisfies the condenser principle.

The condenser potential is constructed as sum of an alternating infinite series. As an application it is proved that the condenser measures are concentrated on the boundaries if and only if $\left(\mu_{t}\right)_{t>0}$ consists of probability measures and is of local type. Results of this type was obtained by Itô [8] for Dirichlet spaces.

A similar approach to condenser potentials but in the context of function kernels has been given by Kishi [9].

In section 3 we finally give an extension of the condenser principle to arbitrary Hunt convolution kernels.

Received March 15, 1977. 


\section{The condenser principle}

Let $G$ be a locally compact abelian group with a fixed Haar measure $\omega_{G}$. In this and the next section $\left(\mu_{t}\right)_{t>0}$ denotes a transient convolution semigroup and $\kappa=\int_{0}^{\infty} \mu_{t} d t$ is the potential kernel. By $D^{+}(\kappa)$ we note the set of positive measures $\mu$ on $G$ for which $\kappa * \mu$ exists.

For a compact neighbourhood $V$ of 0 we always denote by $\varepsilon_{C V}$ the canonical $\kappa$-balayaged measure of $\varepsilon_{0}$ on $C V$. We put $\eta_{V}=a_{V} \kappa *\left(\varepsilon_{0}-\varepsilon_{C V}\right)$ where $a_{V}$ is the uniquely determined number such that $\eta_{V}(G)=1$. Here and in the following we mainly use the terminology from [2].

The existence of condenser potentials is formulated in the following theorem.

THEOREM 1. Condenser principle.

Let $\left(\Omega_{0}, \Omega_{1}\right)$ be a pair of open subsets of $G$ such that $\bar{\Omega}_{0}$ is compact and disjoint from $\bar{\Omega}_{1}$. Then there exist positive measures $\mu_{0}, \mu_{1} \in D^{+}(\kappa)$ such that $\xi=\kappa *\left(\mu_{0}-\mu_{1}\right)$ satisfies

(i) $0 \leq \xi \leq \omega_{G}$,

(ii) $\xi=\omega_{G}$ in $\Omega_{0}$,

(iii) $\xi=0$ in $\Omega_{1}$,

(iv) $\operatorname{supp}\left(\mu_{0}\right) \subseteq \bar{\Omega}_{0}, \operatorname{supp}\left(\mu_{1}\right) \subseteq \bar{\Omega}_{1}$.

Proof. Let $\lambda_{0}$ be the canonical equilibrium distribution for $\Omega_{0}$. Then $0 \leq \kappa * \lambda_{0} \leq \omega_{G}$ and $\kappa * \lambda_{0}=\omega_{G}$ in $\Omega_{0}$ (cf. [2] p. 156). We define a sequence $\left(\lambda_{n}\right)_{n \geq 0}$ of measures inductively: $\lambda_{2 n+1}$ is the canonical balayaged measure of $\lambda_{2 n}$ on $\Omega_{1}$, and $\lambda_{2 n+2}$ is the canonical balayaged measure of $\lambda_{2 n+1}$ on $\Omega_{0}$, $n \geq 0$. We then have for $n \geq 0$

(1) $\kappa * \lambda_{2 n+1} \leq \kappa * \lambda_{2 n}$ with equality in $\Omega_{1}$,

(2) $\kappa * \lambda_{2 n+2} \leq \kappa * \lambda_{2 n+1}$ with equality in $\Omega_{0}$,

(3) $\operatorname{supp}\left(\lambda_{2 n}\right) \subseteq \bar{\Omega}_{0}, \operatorname{supp}\left(\lambda_{2 n+1}\right) \subseteq \bar{\Omega}_{1}$.

The sequence $\left(\kappa * \lambda_{n}\right)$ is decreasing and converges accordingly vaguely to a potential $\kappa * \tau$, and $\tau$ is the vague limit of $\left(\lambda_{n}\right)$ (cf. [2], Th. 16.10). It follows by (3) that $\tau$ must be supported by $\bar{\Omega}_{0} \cap \bar{\Omega}_{1}$ and hence zero. Having shown that $\left(\kappa * \lambda_{n}\right)$ decreases to zero, we get that the infinite alternating series

$$
\sum_{n=0}^{\infty}(-1)^{n} \kappa * \lambda_{n}
$$

is vaguely convergent. 
The $n^{\prime}$ th partial sum and the sum of (4) is denoted respectively $S_{n}$ and $\xi$. By (1) we get

$$
S_{2 n}=\sum_{k=0}^{n-1}\left(\kappa * \lambda_{2 k}-\kappa * \lambda_{2 k+1}\right) \geq 0
$$

and $S_{2 n}=0$ in $\Omega_{1}$, and by (2) we get

$$
S_{2 n+1}=\kappa * \lambda_{0}-\sum_{k=1}^{n}\left(\kappa * \lambda_{2 k-1}-\kappa * \lambda_{2 k}\right) \leq \kappa * \lambda_{0} \leq \omega_{G}
$$

and

$$
S_{2 n+1}=\kappa * \lambda_{0}=\omega_{G} \quad \text { in } \Omega_{0} .
$$

This shows that $0 \leq \xi \leq \kappa * \lambda_{0} \leq \omega_{G}$ and that $\xi=\omega_{G}$ in $\Omega_{0}, \xi=0$ in $\Omega_{1}$.

We finally have to show that $\xi$ has the form $\kappa *\left(\mu_{0}-\mu_{1}\right)$ for measures $\mu_{0}$ and $\mu_{1}$ satisfying (iv). In order to obtain this we actually show that the series (4) is absolutely convergent.

We first prove the following formula

$$
\sum_{n=0}^{\infty}(-1)^{n} \varepsilon_{C V} * \kappa * \lambda_{n}=\varepsilon_{C V} * \xi \quad \text { (vaguely). }
$$

Since the series (4) is alternating we have for $n \geq 0$

$$
0 \leq \xi-S_{2 n} \leq \kappa * \lambda_{2 n}, \quad 0 \leq S_{2 n+1}-\xi \leq \kappa * \lambda_{2 n+1},
$$

and hence

$$
0 \leq \varepsilon_{C V} * \xi-\varepsilon_{C V} * S_{2 n} \leq \varepsilon_{C V} * \kappa * \lambda_{2 n} \leq \kappa * \lambda_{2 n}
$$

and

$$
0 \leq \varepsilon_{C V}^{*} * S_{2 n+1}-\varepsilon_{C V} * \xi \leq \varepsilon_{C V} * \kappa * \lambda_{2 n+1} \leq \kappa * \lambda_{2 n+1},
$$

which show that $\left(\varepsilon_{C V} * S_{n}\right)$ converges vaguely to $\varepsilon_{C V} * \xi$, i.e. that (5) holds.

For every compact neighbourhood $V$ of 0 we finally have

$$
\sum_{n=0}^{\infty}(-1)^{n} \kappa *\left(\varepsilon_{0}-\varepsilon_{C V}\right) * \lambda_{n}=\xi-\varepsilon_{C V} * \xi .
$$

Let now $V$ be so small that $\bar{\Omega}_{0}+V$ is disjoint from $\bar{\Omega}_{1}+V$ and let $\phi \in C_{c}^{+}(G)$ be chosen so that $\phi=1$ on $\bar{\Omega}_{0}+V$ and $\phi=0$ on $\bar{\Omega}_{1}+V$. By (3) we have

$$
\left\langle\kappa *\left(\varepsilon_{0}-\varepsilon_{C V}\right) * \lambda_{2 n+1}, \phi\right\rangle=0,
$$


and

$$
\left\langle\kappa *\left(\varepsilon_{0}-\varepsilon_{C V}\right) * \lambda_{2 n}, \phi\right\rangle=\frac{1}{a_{V}} \lambda_{2 n}(G),
$$

so by (6)

$$
\frac{1}{a_{V}} \sum_{n=0}^{\infty} \lambda_{2 n}(G)=\left\langle\xi-\varepsilon_{C V} * \xi, \phi\right\rangle\langle\infty .
$$

This implies that the series $\sum_{n=0}^{\infty} \lambda_{2 n}$ is vaguely convergent with sum $\mu_{0}$, which is a positive measure supported by the compact set $\bar{\Omega}_{0}$. We clearly have

$$
\kappa * \mu_{0}=\sum_{n=0}^{\infty} \kappa * \lambda_{2 n},
$$

so by (4) the sequence

$$
\kappa *\left(\sum_{k=0}^{n} \lambda_{2 k+1}\right)
$$

increases to $\kappa * \mu_{0}-\xi$. By Lemma 15.7 of [2] follows that the series $\sum_{k=0}^{\infty} \lambda_{2 k+1}$ is vaguely convergent, and the sum $\mu_{1}$ is a positive measure supported by $\bar{\Omega}_{1}$. By Lemma 15.3 of [2] we finally get that $\mu_{1} \in D^{+}(\kappa)$ and $\kappa * \mu_{1}=\kappa * \mu_{0}-\xi$. $\square$

Remarks. 1) It follows from the above proof that $\mu_{0}, \mu_{1}$ and $\xi$ are canonically constructed and that

$$
\xi=\sum_{n=0}^{\infty}(-1)^{n} \kappa * \lambda_{n},
$$

where $\lambda_{0}$ is the canonical equilibrium distribution for $\Omega_{0}, \lambda_{2 n+1}$ is the canonical balayaged measure of $\lambda_{2 n}$ on $\Omega_{1}$ and $\lambda_{2 n+2}$ is the canonical balayaged measure of $\lambda_{2 n+1}$ on $\Omega_{0}$. Furthermore

$$
\mu_{0}=\sum_{n=0}^{\infty} \lambda_{2 n}, \quad \mu_{1}=\sum_{n=0}^{\infty} \lambda_{2 n+1} .
$$

The measure $\mu_{0}$ (resp. $\mu_{1}$ ) is called the inner (resp. outer) canonical condenser distribution, and $\xi=\kappa *\left(\mu_{0}-\mu_{1}\right)$ is called the canonical condenser potential for the pair $\left(\Omega_{0}, \Omega_{1}\right)$.

2) The canonical condenser potential for the pair $\left(\Omega_{0}, \Omega_{1}\right)$ is dominated by the canonical equilibrium potential for $\Omega_{0}$, i.e. $\xi \leq \kappa * \lambda_{0}$. If 
$\Omega_{1}=\phi$ they are equal.

3) Since $\kappa$ satisfies the principle of positivity of mass (cf. [2] Th. 16.25) we have $\mu_{1}(G) \leq \mu_{0}(G)$.

Under certain further assumptions we have $\mu_{0}(G)=\mu_{1}(G)$, see Corollary 4 below.

\section{Applications}

We recall that $\left(\mu_{t}\right)_{t>0}$ is called of local type if its infinitesimal generator on $C_{0}(G)$ is a local operator. This is equivalent with $\kappa$ satisfying the principle of local unicity of mass: If $\kappa * \sigma_{1}=\kappa * \sigma_{2}$ in an open set $U$ then $\sigma_{1}=\sigma_{2}$ in $U$. It is also equivalent with $\operatorname{supp}\left(\varepsilon_{C V}\right) \subseteq \partial V$ for all (or sufficiently small) neighbourhoods $V$ of 0 . For these and other equivalent properties see [2] $\S 18$.

We can formulate an equivalent condition in terms of the condenser distributions.

THEOREM 2. Let $\kappa$ be a potential kernel for a transient convolution semigroup $\left(\mu_{t}\right)_{t>0}$. Then $\left(\mu_{t}\right)_{t>0}$ is of local type if and only if the following holds:

For any pair of open sets $\left(\Omega_{0}, \Omega_{1}\right)$ for which $\bar{\Omega}_{0}$ is compact and disjoint from $\bar{\Omega}_{1}$ the canonical outer condenser distribution $\mu_{1}$ is supported by $\partial \Omega_{1}$.

Proof. Suppose first that $\left(\mu_{t}\right)_{t>0}$ is of local type. Since $\kappa * \mu_{0}=\kappa * \mu_{1}$ in $\Omega_{1}$ we get by the principle of local unicity of mass that $\mu_{0}=\mu_{1}$ in $\Omega_{1}$, and since $\operatorname{supp}\left(\mu_{0}\right) \cap \bar{\Omega}_{1}=\phi$ we have $\mu_{1}\left(\Omega_{1}\right)=\mu_{0}\left(\Omega_{1}\right)=0$, hence $\operatorname{supp}\left(\mu_{1}\right)$ $\subseteq \partial \Omega_{1}$.

Conversely, suppose that the outer condenser distribution $\mu_{1}$ always lies on the boundary of $\Omega_{1}$. The measure $\check{\kappa}$ is potential kernel for the reflected semigroup $\left(\check{\mu}_{t}\right)_{t>0}$, and it suffices to prove that $\left(\check{\mu}_{t}\right)_{t>0}$ is of local type. Let $V$ be a compact neighbourhood of 0 in $G$ and let $\delta_{C V}$ denote the canonical $\check{\kappa}$-balayaged measure of $\varepsilon_{0}$ on $C V$. Then $\check{\kappa} * \delta_{C V} \leq \check{\kappa}$ with equality in $C V$. It suffices to prove that $\delta_{C V}(C V)=0$. Let $\Omega_{0}$ be an open set such that $\bar{\Omega}_{0}$ is compact and contained in $C V$. We choose an open set $\Omega_{1} \supseteq V$ such that $\bar{\Omega}_{1} \cap \bar{\Omega}_{0}=\phi$ and let $\xi=\kappa *\left(\mu_{0}-\mu_{1}\right)$ be the canonical condenser potential for the pair $\left(\Omega_{0}, \Omega_{1}\right)$.

For an approximate unit $\left(\phi_{i}\right)_{i \in I}$ from $C_{c}^{+}(G)$ for which the support of $\phi_{i}$ is sufficiently close to 0 for all $i \in I$ we have $\left(\mu_{0}-\mu_{1}\right) * \phi_{i}=0$ on $V$, 
because $\operatorname{supp}\left(\mu_{0}\right) \subseteq \bar{\Omega}_{0}$ and $\operatorname{supp}\left(\mu_{1}\right) \subseteq \partial \Omega_{1}$. Therefore we have

$$
\left\langle\delta_{C V}, \xi * \phi_{i}\right\rangle=\left\langle\check{\kappa} * \delta_{C V},\left(\mu_{0}-\mu_{1}\right) * \phi_{i}\right\rangle=\left\langle\breve{\kappa},\left(\mu_{0}-\mu_{1}\right) * \phi_{i}\right\rangle=\xi * \phi_{i}(0) .
$$

Given a compact subset $A \subseteq \Omega_{0}$ we have $\xi * \phi_{i}=1$ on $A$ and $\xi * \phi_{i}(0)$ $=0$ if $\operatorname{supp}\left(\phi_{i}\right)$ is sufficiently close to 0 , so we have $\delta_{C V}(A)=0$. By the arbitrariness of $A$ we get $\delta_{C V}\left(\Omega_{0}\right)=0$ and by the arbitrariness of $\Omega_{0}$ we get $\delta_{C V}(C V)=0$. $\quad$ o

It is also possible to give necessary and sufficient conditions that both inner and outer condenser distributions lie on the boundary.

We shall use below that $\mu_{t}(G)=1$ for one (or all) $t>0$ if and only if $\varepsilon_{C V}(G)=1$ for one (or all) $V$. (Cf. e.g. [4] Th. 5).

It will be convenient with the following Proposition.

Proposition 3. Let $\kappa$ be a potential kernel for a transient convolution semigroup $\left(\mu_{t}\right)_{t>0}$ of probability measures. If $\kappa * \mu=\kappa * \nu$ in the complement of some compact set, then $\mu(G)=\nu(G)$.

Proof. For an open relatively compact set $\Omega$ we denote by $\lambda_{\Omega}$ the canonical equilibrium distribution for $\Omega$. Then $\kappa * \lambda_{\Omega}$ tends vaguely to $\omega_{G}$ as $\Omega$ increases to $G$. Since $\kappa * \lambda_{\Omega} \leq \omega_{G}$ we get that $\varepsilon_{C V} * \kappa * \lambda_{\Omega}$ converges vaguely to $\varepsilon_{C V} * \omega_{G}=\omega_{G}$ and hence that $\eta_{V} * \lambda_{\Omega}$ converges vaguely to 0 as $\Omega$ increases to $G$. This implies that $\lambda_{\Omega}$ converges vaguely to 0 .

Let $\phi \in C_{c}^{+}(G)$ be symmetric of integral 1. Then there exists a function $f \in C_{c}^{+}(G)$ such that $\kappa * \mu * \phi \leq \kappa * \nu * \phi+f$ and hence

$$
\begin{aligned}
\mu(G)= & \lim _{\Omega \rightarrow G} \kappa * \lambda_{\Omega} * \mu * \phi(0) \leq \lim _{\Omega \cap G} \kappa * \lambda_{\Omega} * \nu * \phi(0) \\
& +\lim _{\Omega \cap G} \lambda_{\Omega} * f(0)=\nu(G) .
\end{aligned}
$$

Similarly we get $\nu(G) \leq \mu(G)$. व

COROLLARY 4. Let $\kappa$ be a potential kernel for a transient convolution semigroup of probability measures.

(i) For every positive measure $\mu \in D^{+}(\kappa)$ and every open set $\Omega$ for which $C \Omega$ is compact we have $\mu^{\circ}(G)=\mu(G)$, where $\mu^{\Omega}$ is the canonical balayaged measure of $\mu$ on $\Omega$.

(ii) For any pair $\left(\Omega_{0}, \Omega_{1}\right)$ of open sets for which $\bar{\Omega}_{0}$ is compact and $\Omega_{1}$ is the complement of a compact neighbourhood of $\bar{\Omega}_{0}$, the condenser measures $\mu_{0}$ and $\mu_{1}$ for $\left(\Omega_{0}, \Omega_{1}\right)$ have same total mass: $\mu_{0}(G)=\mu_{1}(G)$. 
Proof. (i) follows since $\kappa * \mu=\kappa * \mu^{\Omega}$ in $\Omega$. (ii). With the terminology of the proof of Theorem 1 we have by (i) that $\lambda_{2 n+1}(G)=\lambda_{2 n}(G)$ for all $n \geq 0$ and hence $\mu_{0}(G)=\mu_{1}(G)$. $\quad$

THEOREM 5. Let $\kappa$ be a potential kernel for a transient convolution semigroup $\left(\mu_{t}\right)_{t>0}$. Then $\left(\mu_{t}\right)_{t>0}$ is of local type and $\mu_{t}(G)=1$ for all $t>0$ if and only if the following holds:

For any pair of open sets $\left(\Omega_{0}, \Omega_{1}\right)$ for which $\bar{\Omega}_{0}$ is compact and disjoint from $\bar{\Omega}_{1}$ the condenser distributions $\mu_{0}$ and $\mu_{1}$ are supported by $\partial \Omega_{0}$ and $\partial \Omega_{1}$ respectively.

Theorem 5 follows from the following two propositions together with Theorem 2.

Proposition 6. Let $\kappa$ be the potential kernel for a transient convolution semigroup $\left(\mu_{t}\right)_{t>0}$ of local type and such that $\mu_{t}(G)=1$ for all $t>0$.

If $\kappa * \mu=\kappa * \nu+\omega_{G}$ in an open set $\Omega$ then $\mu=\nu$ in $\Omega$.

Proof. Let $f \in C_{c}^{+}(\Omega)$ and choose a compact neighbourhood $V_{0}$ of 0 such that $\operatorname{supp}(f)-V_{0} \subseteq \Omega$. For $V \subseteq V_{0}$ we then have $\check{\varepsilon}_{C V} * f \in C_{c}^{+}(\Omega)$ because $\operatorname{supp}\left(\varepsilon_{C V}\right) \subseteq \partial V$, and therefore

$$
\left\langle\kappa * \mu, f *\left(\varepsilon_{0}-\check{\varepsilon}_{C V}\right)\right\rangle=\left\langle\kappa * \nu, f *\left(\varepsilon_{0}-\check{\varepsilon}_{C V}\right)\right\rangle+\left\langle\omega_{G}, f *\left(\varepsilon_{0}-\check{\varepsilon}_{C V}\right)\right\rangle,
$$

but the last term vanishes because $\varepsilon_{C V}(G)=1$. We then have

$$
\left\langle\eta_{V} * \mu, f\right\rangle=\left\langle\eta_{V} * \nu, f\right\rangle
$$

and for $V \rightarrow 0$ we get $\langle\mu, f\rangle=\langle\nu, f\rangle$, hence $\mu=\nu$ in $\Omega$.

Proposition 7. Let $\kappa$ be a potential kernel for a transient convolution semigroup $\left(\mu_{t}\right)_{t>0}$.

If the canonical equilibrium distribution $\lambda_{0}$ for an arbitrary open relatively compact set $\Omega_{0}$ is supported by $\partial \Omega_{0}$, then $\mu_{t}(G)=1$ for all $t>0$.

Proof. It suffices to prove that $\varepsilon_{C V}(G)=1$ for all sufficiently small compact neighbourhoods $V$ of 0 . Let $\Omega_{0}$ be a non-empty open relatively compact set and let $f \in C_{c}^{+}\left(\Omega_{0}\right)$ be such that $\left\langle\omega_{G}, f\right\rangle=1$. Denoting by $\lambda_{0}$ the canonical equilibrium distribution for $\Omega_{0}$, we have $\operatorname{supp}\left(\lambda_{0}\right) \subseteq \partial \Omega_{0}$.

For $V$ so small that $\operatorname{supp}(f)-V \subseteq \Omega_{0}$ we therefore have

$$
0=\left\langle\lambda_{0}, \check{\kappa} *\left(\varepsilon_{0}-\check{\varepsilon}_{C V}\right) * f\right\rangle=\left\langle\kappa * \lambda_{0}, f-\check{\varepsilon}_{C V} * f\right\rangle,
$$


and hence

$$
1=\left\langle\kappa * \lambda_{0}, f\right\rangle=\left\langle\kappa * \lambda_{0}, \check{\varepsilon}_{C V} * f\right\rangle \leq\left\langle\omega_{G}, \check{\varepsilon}_{C V} * f\right\rangle=\varepsilon_{C V}(G) .
$$

On the other hand we always have $\varepsilon_{C V}(G) \leq 1$.

\section{Extension to Hunt convolution kernels}

In this section we drop the assumption that $\mu_{t}(G) \leq 1$ for all $t>0$ and suppose $\kappa=\int_{0}^{\infty} \mu_{t} d t$ is an arbitrary Hunt convolution kernel on $G$, cf. [6]. For an excessive measure $\tau$ on $G$ (i.e. $\mu_{t} * \tau \leq \tau$ for all $t>0$ ) and an open set $\Omega$ we define the reduced measure $R_{\tau}^{\Omega}$ as the infimum of all excessive measures majorizing $\tau$ on $\Omega$. The reduced measure $R_{\tau}^{\Omega}$ is again excessive, and if $\Omega$ is relatively compact $R_{\tau}^{\Omega}$ is a potential $R_{\tau}^{\Omega}=\kappa * \lambda$ generated by a uniquely determined measure $\lambda \in D^{+}(\kappa)$, cf. [4].

By the same method of proof as in Theorem 1 we obtain the following generalization of Theorem 1 :

THEOREM 8. Let $\kappa$ be a Hunt convolution kernel and let $\tau$ be an excessive measure. For any pair $\left(\Omega_{0}, \Omega_{1}\right)$ of open sets such that $\bar{\Omega}_{0}$ is compact and disjoint from $\bar{\Omega}_{1}$ there exist positive measures $\mu_{0}, \mu_{1} \in D^{+}(\kappa)$ such that $\xi=\kappa *\left(\mu_{0}-\mu_{1}\right)$ satisfies

(i) $0 \leq \xi \leq \tau$,

(ii) $\xi=\tau$ in $\Omega_{0}$,

(iii) $\xi=0$ in $\Omega_{1}$,

(iv) $\operatorname{supp}\left(\mu_{0}\right) \subseteq \bar{\Omega}_{0}, \operatorname{supp}\left(\mu_{1}\right) \subseteq \bar{\Omega}_{1}$.

The measure $\xi$ is sum of the infinite alternating series

$$
\xi=\sum_{n=0}^{\infty}(-1)^{n} \kappa * \lambda_{n},
$$

where $\lambda_{0}$ is the uniquely determined measure such that $R_{\tau}^{\Omega_{0}}=\kappa * \lambda_{0}, \lambda_{2 n+1}$ is the canonical balayaged measure of $\lambda_{2 n}$ on $\Omega_{1}$ and $\lambda_{2 n+2}$ is the canonical balayaged measure of $\lambda_{2 n+1}$ on $\Omega_{0}, n \geq 0$. Furthermore

$$
\mu_{0}=\sum_{n=0}^{\infty} \lambda_{2 n} \quad \text { and } \quad \mu_{1}=\sum_{n=0}^{\infty} \lambda_{2 n+1} .
$$

Remark. Theorem 1 is obtained for $\tau=\omega_{G}$ which is excessive if and only if $\mu_{t}(G) \leq 1$ for all $t>0$, i.e. if and only if $\kappa$ is the potential kernel for a transient convolution semigroup in the above sense. 


\section{REFERENCES}

[1] Berg, C., Forst, G.: Non-symmetric translation invariant Dirichlet forms. Invent. Math. 21 (1973), 199-212.

[2] — : Potential theory on locally compact abelian groups. Ergebnisse der Mathematik Bd. 87. Berlin Heidelberg New York: Springer 1975.

[ 3 ] Bliedtner, J.: Dirichlet forms on regular functional spaces. Seminar on potential theory, II. Lecture Notes in Mathematics vol. 226. Berlin Heidelberg New York: Springer 1971.

[ 4 ] Deny, J.: Familles fondamentales, Noyaux associés. Ann. Inst. Fourier 3 (1951), 73-101.

[5] _ : Sur les espaces de Dirichlet. Sém. de Théorie du Potentiel, Paris, Ire année, 1957.

[6] — : Noyaux de convolution de Hunt et noyaux associés a une famille fondamentale. Ann. Inst. Fourier 12 (1962), 643-667.

[ 7 ] _ : Méthodes hilbertiennes en théorie du potentiel. Potential Theory (C.I.M.E. I Ciclo, Stresa) 121-201. Rome: Ed. Cremonese 1970.

[8] Itô, M.: Characterizations of supports of balayaged measures. Nagoya Math. J. 28 (1966), 203-230.

[9] Kishi, M.: Sur l'existence des mesures des condensateurs. Nagoya Math. J. 30 (1967), 1-7.

[10] Landkof, N. S.: Foundations of modern potential theory. Die Grundlehren Bd. 180. Berlin Heidelberg New York: Springer 1972.

Matematisk Institut, Kobenhavns Universitet

Universitetsparken 5

2100 Copenhagen $\phi$

Denmark 